\title{
Automatic Generation of Dynamic Simulation Models based on Standard Engineering Data
}

\author{
Niklas Paganus $^{1} \quad$ Marko Luukkainen $^{2} \quad$ Karri Honkoila $^{1}$ Tommi Karhela ${ }^{2}$ \\ ${ }^{1}$ Fortum Power and Heat Ltd., Finland, \{niklas . paganus, karri.honkoila\}@for tum.com \\ ${ }^{2}$ VTT Technical Research Centre of Finland Ltd., Finland, \{marko. luukkainen, tommi.karhela\}@vtt.fi
}

\begin{abstract}
Dynamic process simulation is used to mitigate risks, reduce costs and improve quality of design in plant engineering. Traditionally, simulation models are created manually from engineering source data. Benefits of utilising simulation are recognised by the industry, but simulation is not exploited to its full potential due to its current laborious nature. Engineering software interoperability improves efficiency in engineering workflows. Required manual work is reduced, enabling faster and more robust design to be conducted. In this paper, work conducted by authors in integrating the dynamic process simulation software Apros into the engineering workflow by automatically creating simulation models based on standard engineering data is reported. A case study was conducted to demonstrate the implemented features. Process engineering data in the Proteus XML format was used as the source data for simulation model generation. The case study shows that the implemented features reduce manual work required, lowering the threshold for utilising simulation.
\end{abstract}

Keywords: simulation, engineering workflow, interoperability, virtual plant

\section{Introduction}

Efficiency of engineering actions in plant design is wanted to be improved in process industry and power generation. Profitable and safe operations are wanted to be achieved faster. Advanced computer aided engineering (CAE) tools are utilised in the engineering workflow, allowing engineers to conduct their work efficiently. At the same time engineering projects struggle with delays and costs related to correction of design flaws recognised late in the engineering workflow or during the operation of a plant.

Two alternatives for solving these issues are discussed in this paper. First, a wider and earlier utilisation of dynamic process simulation can help in identifying engineering errors earlier, reducing or avoiding costs of corrective actions. Second, engineering software interoperability enables engineers from different disciplines to work in a more integrated manner, hence improving communication in the workflow. Thereby, time and costs required for engineering are reduced and sources for engineering errors are prevented. Additionally in this paper, it is shown how engineering software interoperability lowers the threshold for utilising dynamic process simulation by enabling automatic generation of simulation models.

Plant engineering actions are typically organised as an engineering workflow. Practices in the industry vary, but in common practises the actions are organised into design phases in which different engineering disciplines conduct their design effort. The focus disciplines of this paper are process, automation and simulation engineering.

Process engineers conduct their design effort using CAE software, resulting in piping and instrumentation diagrams (P\&ID) and 3D models describing the plant. Typically, the basic design is delivered as P\&IDs and the detailed design is implemented with 3D models. Typical engineering software use proprietary data models and file formats for representing design data instead of exploiting standards. This also applies to offered interfaces available in software. This restricts software interoperability and thereby both resource intensive and error-prone manual information transfer is taking place in the engineering workflow (Karhela et al, 2012; Estevez et al, 2012). However, an emerging trend of utilising standard data models and formats is emerging (Estevez et al, 2012), enabling engineering software interoperability. The ISO 15926 standard (International Organization for Standardization, 2004) and the related Proteus XML specification (Fiatech and POSC Caesar Association, 2016) are examples of standardisation being adopted by the industry. Such data formats serve as proper initial data also for extended use cases, e.g. for generating simulation models as exploited in this paper.

Simulation is utilised in the engineering workflow to answer engineering and operational questions with lower risks compared to traditional testing (Oppelt et al, 2015a). The dynamic behaviour of a plant can be analysed using dynamic process simulation. Simulation is not utilised to its full capability due to its laborious modelling requirement, partly as a result of noninteroperable engineering software (Karhela et al, 2012; Oppelt et al, 2014). Modelling effort can be significantly 
reduced by automating simulation model generation based on available design data (Karhela et al, 2012). In practise this means that simulation software must be made interoperable with plant engineering software. This can be achieved by implementing customised software interfaces, but committing to standards makes the interfaces reusable. Highest efficiency can be achieved if simulation models can be generated based on the same standard engineering data as CAE software use for standard engineering data transfer.

In this paper the effort made by the authors in developing automatic simulation model generation based on standard engineering data is reported. The feature has been developed for dynamic process simulation software Apros 6, which utilises the Simantics platform. In the case study reported in this paper, a simulation model is created based on P\&ID source data. The P\&ID is drawn in Intergraph SmartPlant PID and exported into the ISO 15926-based Proteus XML format. An Apros model was generated based on a predefined ruleset.

The paper is structured in the following way. After this introductory section, relevant related research is presented in section 2 . In section 3 , the work conducted by the authors for Apros simulation software and the Simantics platform is reported. In section 4, a small case study conducted for this paper to demonstrate the implemented features is reported. Finally, the paper is concluded in section 5 .

\section{Related work}

In this section, previous research relevant for this paper is reviewed and necessary terminology is defined. Major topics are the plant engineering workflow, engineering software interoperability and the role of simulation in plant engineering with focus on simulation model generation.

In this paper the concept plant engineering workflow is used to describe the organisation of engineering actions in a typical plant construction or retrofitting project. The concept is simplified from actual workflows in use since industry practises vary significantly. Thereby, a generalised workflow is formulated. Similar concepts used in literature are e.g. the plant engineering process (Hoyer et al, 2005), the design process (Towler et al, 2013) and the lifecycle of a process plant (Oppelt et al, 2015a). In the workflow, engineering actions are divided into design phases and participating engineering disciplines. The generalised workflow used in this paper is assumed to consist of five phases. These phases are 1. Conceptual design, 2. Basic design, 3. Detailed design, 4. Commissioning, 5. Operation and maintenance. Most relevant phases for this paper are the basic and detailed design phases.

The engineering workflow requires seamless cooperation between engineering disciplines. Engineers are accustomed to using best-in-class engineering software and interoperability has been restricted by lacking interfaces of software tools. Design data is handed over both within and across disciplines as the workflow proceeds. Design information is lost in the transfers when non-interoperable engineering software is used and significant manual action is required. The emerging trend of engineering software interoperability and utilisation of standards for representing design data will improve work conducted in the workflow as transferring design data can be automated. This requires that engineering software tools are equipped with standard interfaces to allow engineers to continue using their preferred tools.

A few possible alternatives for standard engineering data representation exist. The ISO 15926 standard (International Organization for Standardization, 2004) is being adopted by the process industry and in power plant engineering as a neutral data format. Originally, the standard did not define an actual data transfer format and for this purpose the XMpLant (Nextspace, 2015) Extensive Markup Language (XML)-based data format was developed for ISO 15926 data and was adopted widely in the industry. The XMpLant schema was made public and afterwards it has been developed by Fiatech (Fiatech, 2009) under the Proteus name. Thereby, this format is called Proteus XML. In a more recent part 8 of the ISO 15926 standard, a Web Ontology language (OWL)-based data format is defined, but it has not gained wide acceptance in industry. The Proteus XMLformat allows representation of both P\&ID and 3D data and contains both the geometry and attribute information in the same XML-file. Several commercial CAE tools aimed for P\&ID and 3D design support Proteus or the related XMpLant format. Proteus is being actively developed by Fiatech in its IIMM project (Fiatech, 2015) and in other standardisation initiatives such as the DEXPI (DEXPI, 2016).

CAEX (Computer Aided Engineering Exchange) defined in IEC 62424 (International Electrotechnical Commission, 2008) is an engineering data exchange format that can be used for vendor independent data exchange, e.g. for P\&IDs. CAEX has been used for generating simulation models (Hoernicke et al, 2015; Barth et al, 2009) and is also utilised by AutomationML. (Holm et al, 2012) The format has not been adopted by the industry as widely as ISO 15926 and it lacks an established industrial reference data library similar to the one available for ISO 15926.

ISO 10303 (International Organization for Standardization, 1994), or commonly known as STEP (Standard for the Exchange of Product model data) has been used to integrate manufacturing systems in several industries (Tursi et al, 2009), but it has not been adopted in process industry and power generation. ISO 15926 can be considered as a successor to STEP in process industries (Wiesner et al, 2011). 
IFC (Industry Foundation Classes), also published as ISO 16739 standard (International Organization for Standardization, 2013), is a neutral data format maintained by buildingSMART International (buildingSMART International, 2016). It has been widely adopted in construction industry. IFC can be used to represent e.g. technical systems in buildings, such as heating and water piping, but it is not aimed for representing industrial processes and lacks component libraries for such processes. Therefore, ISO 15926 currently outrules IFC when considering needs for industrial simulation model generation. IFC is though prominent and could possibly be used partly as source data in 3D and future extensions might improve usability for industrial processes.

Dynamic process simulation is utilised in the engineering workflow to answer questions related to the dynamic behaviour of a plant. Conditions too costly or dangerous to test with traditional testing methods can be tested. In industry, despite recognised benefits of utilising simulation, it is commonly exploited only at certain times when needed and usually very late in the engineering workflow (Karhela et al, 2012). Corrective actions are more expensive and cause more disturbances to project schedules compared to if errors would have been discovered and corrected in an earlier phase (Oppelt et al, 2015b; Barth et al, 2013). Therefore, ways to support utilisation of simulation more extensively and earlier in the engineering workflow are needed to promote utilisation of simulation and gaining corresponding benefits.

Simulation models have traditionally been modelled manually based on process design data such as P\&IDs and isometric drawings as printouts and by manually inspecting data from 3D models. Automation functionalities are added to the model based on automation diagrams. Simulation modelling therefore consists of combining heterogeneous data (Barth et al, 2013). Modelling work required for creating simulation models is considered laborious (Karhela et al, 2012) and research related to automating simulation model generation is actively conducted. See e.g. (Hoyer et al, 2005), (Hoernicke et al, 2015), (Barth et al, 2009), (Barth et al, 2013) and (Laakso et al, 2013) for previous work in automatic simulation model generation. P\&IDs and other design documents are nowadays intelligent since CAE tools exploit object oriented features. Therefore every component on a P\&ID has both its graphical appearance and attribute data defined (Barth et al, 2013), serving as a proper source for simulation model generation. By utilising automatic simulation model generation the models can be created faster, more accurate and the method is also less error-prone than manual modelling. These benefits should be enough to improve the profitability of simulation and thereby lowering the threshold for utilising simulation. This can enable simulation to be become a more integrated part of every phase of the engineering workflow.

Many previous proposals and implementations for automatic simulation model generation have been created by interfacing two specific software resulting in a custom integration. Tool specific approaches have also been implemented for Apros previously (Laakso et al, 2013; Paljakka et al, 2009). Standard interfaces allow implementation of reusable features. Standards being adopted in plant design should also be utilised when creating simulation models. The ISO 15926-based Proteus XML-format is promising as source data for automatic simulation model generation since it allows representation of both P\&ID and 3D data, describes both the geometry and attribute information and is supported by major CAE software.

\section{Implementation}

The authors have implemented features for automatic simulation model generation based on Proteus XML data in Apros and Simantics software environment. First, the software environment is presented followed by the description of the implemented features.

Apros (Fortum and VTT Technical Research Centre of Finland, 2016) is a simulation software for modelling and simulation of dynamic processes developed and offered by Fortum and VTT Technical Research Centre of Finland since 1986. Apros has mostly been utilised in modelling and simulation of nuclear and combustion power plants with new application areas emerging. The core feature of Apros is the thermal hydraulic solver for one-dimensional two-phase flow (Porkholm et al, 2016). Apros offers an extensive library of process, automation and electrical components for modelling industrial processes. Recently, Apros has been integrated with automation engineering by introducing features for transferring automation design data in standard format to detailed design from Apros (Paganus et al, 2016). The newest version, Apros 6, is based on the Simantics platform.

Simantics (Simantics, 2016) is an open ontologybased integration platform for modelling and simulation (Karhela et al, 2012). It is managed by the THTH Association of Decentralized Information Management for Industry (THTH Association of Decentralized Information Management for Industry, 2016). Simantics offers a semantic triplestore database and a user interface based on Eclipse (The Eclipse Foundation, 2016) technology for its products. In the Apros case, the Apros solver is connected to Simantics, creating a modelling and simulation environment. A dedicated language for manipulating the Simantics database and its plug-ins has been developed, named Simantics Constraint Language (SCL) (Karhela et al, 2012). SCL can be used to perform model transformations, which 
has been utilised by the authors for generating Apros simulation models.

The main objective of the work conducted by the authors was to achieve process simulation integration by exploiting standards for maximising usability in industrial projects, were a wide range of different software and practises are present. As the main source data for automatic generation of Apros models, P\&IDs and $3 \mathrm{D}$ models were used. 3D undoubtedly gives more accurate description of the process than P\&IDs and is the source for detailed accuracy in a simulation model. However, as simulation is wanted to be utilised in an earlier design phase, authors have designed a workflow where the initial simulation model is generated based on P\&IDs. The initial model is made more detailed from 3D data when the source data is available.

The authors have developed a toolset for the Simantics platform for handling Proteus P\&ID and 3D data in XML-format. The data import is based on automatic conversion of XML schemas to Simantics Layer0-based ontologies. Layer0 is the ontology description language used by Simantics (Karhela et al, 2012). Schema conversion tool converts XML element, complex type, and simple type definitions to Layer0 types and creates type specific relations based on XML indicators. For file import purposes, we automatically generate Java classes for SAX-based (SAX, 2004) XML parser, which processes Proteus XML files and creates respective instances into Simantics database.

We developed both P\&ID and 3D visualisations of Proteus data. Proteus format uses STEP (ISO 10303) (International Organization for Standardization, 1994) standard's graphical definitions and Proteus files contain full graphical representation of the original design. Proteus P\&IDs use only few graphical primitives; lines, circles, ellipses, arcs of the latter two, and text fields, making visualising P\&IDs straightforward using Simantics diagramming component and Java2D (Oracle, 2016) graphics layer. $3 \mathrm{D}$ visualisation uses the Visualization Toolkit (VTK, 2016) and OpenCASCADE (Open CASCADE Technology, 2016). The P\&ID visualisation is also used for defining the scope of the data to be transformed into Apros models. The selection is done by painting parts of the diagram, similarly to any raster graphics editor software including freehand drawing tool.

From the P\&ID or 3D data imported into Simantics, Apros simulation models can be generated based on a predefined mapping ruleset from the selections made in the Proteus visualisation. The ruleset was implemented using SCL transformations, since the process is a model transformation were the Proteus data model is transformed into the Apros data model. Mappings can be of one to one, one to many or many to one type. The SCL transformation framework supports all of these mapping options. The basic feature is that equipment and components available in the Proteus data are mapped to Apros components. Attribute data available in the source data is utilised when the simulation model is parametrised. Also, different attributes needed for Apros can be calculated from the process data. Calculations are particularly relevant when an accurate 3D model is available and e.g. piping geometry can be used for calculating parameters necessary for a detailed simulation model. For attributes, the units used are also mapped to be suitable for Apros. The mappings can be altered by the user for specific needs.

The ruleset for generating Apros models has been implemented in a two-level architecture. The base of the ruleset is the reusable general features that handle the common features in Proteus, e.g. connections between components. In each separate project, an additional ruleset is defined according to the needs of the engineering project. This additional ruleset is designed to be very easy and fast to implement: it only requires definition of the corresponding component or attribute type in Proteus and Apros. By implementing this solution, the effort for taking automatic simulation model generation into use is minimised while still allowing project specific modifications. After the initial definition, the ruleset is reusable within the project.

When design and P\&IDs are updated, the changes must be reflected to the simulation model. The challenge is that the user is usually required to make manual changes to the automatically generated model because of missing data and simulator specific needs. Those changes shall not be overridden. Our initial implementation used two way comparison, automatically generated simulation model with user made changes, and simulation model generated from updated P\&IDs. This model does not allow automatic detection of user made changes, so to fix the situation, we have decided to use three-way comparison of the simulation model, one version as the originally generated model, one with user made changes, and one generated from updated P\&IDs. Simantics database versioning capabilities (Karhela et al, 2012) allows for detecting changes that the user has made to the simulation model after it has been generated for the first time. Hence we do not need to store the original model and the model with user made changes separately. This reduces amount of data needed to be stored in the database.

Achievable accuracy of automatically generated simulation models depends heavily on the quality of the source data. During implementation it was concluded that the quality of the Proteus XML data produced by different software varies significantly. This partly relates to the constant development of the Proteus schema, creating a challenge for software vendors to keep up the pace. One example of this is the support for instrumentation. It is evident that the instrumentation support is not yet mature for full-scale industrial projects in most software tools, although basic features 
are covered. The instrumentation model is being revised by Fiatech and DEXPI, which will also require revisions by software vendors.

\section{Case study}

A case study was conducted for this paper to test and validate the Proteus features developed for Apros and Simantics described in section 3 . The scope of the study was to generate an Apros simulation model based on a simple P\&ID. The P\&ID was drawn in Intergraph SmartPlant PID software and then exported into the Proteus XML format. After importing the Proteus data into Simantics, an Apros simulation model was generated.

The example process in this paper is a simple pumptank model. It consists of a water filled tank, TA-113, which is fed with water by activating the pump PU-112. The liquid level in the tank is controlled by adjusting the outward flow with a control valve in the outflow pipeline from the tank. This control requirement has also been drawn into the P\&ID. The P\&ID is illustrated in Figure 1.

The P\&ID in Proteus XML format was imported into Simantics for inspection. The diagram was visualised correctly and the structure and attributes of the diagram can be reviewed by the user. By using the Proteus selection tool in Simantics, the scope intended for Apros model generation was chosen. In this case, the whole diagram is of interest and therefore the full content of the diagram was selected for model generation.

Mapping rules were defined to describe the correspondence between the source P\&ID and Apros model. The general Proteus rule set, described in section 3 , was used as a base and extended according to the specific needs of the case. The specific rules map components, attributes and specify how the unique identifiers are defined in the source data. Some simplifications were made, e.g. the impulse line and its valves have been modelled with a single level measurement component.

Apros model generation is automatic after the mapping rules have been defined. The generated simulation model is illustrated in Figure 2. Both the layout of the diagram and the data was kept consistent and attributes were transformed correctly. After model creation and setting necessary additional parameters for the model, dynamic simulation with initial values can be started. The model generated behaved as expected and controller tuning was successfully conducted. Thereby, the model was equivalent to a manually modelled model, but modelling effort was reduced. The benefit is that the model structure and initial parametrisation can be achieved faster and with fewer errors compared to manual modelling. Drawbacks of the method are that occasionally component and pipeline placements taken from the process data might not be optimal for the simulation model and some components might need to be moved after model generation. Also, many detailed modelling tasks are hard to automate and the degree of automation in model generation should be properly decided.

\section{Conclusions}

Benefits of utilising simulation are recognised by the industry but due to its laborious modelling effort required, dynamic process simulation is not utilised to its full potential in the engineering workflow. Laborious and error-prone manual work can be effectively reduced by automating creation of simulation models. This lowers the threshold for utilising simulation, making it



Figure 1. P\&ID of the case study. 


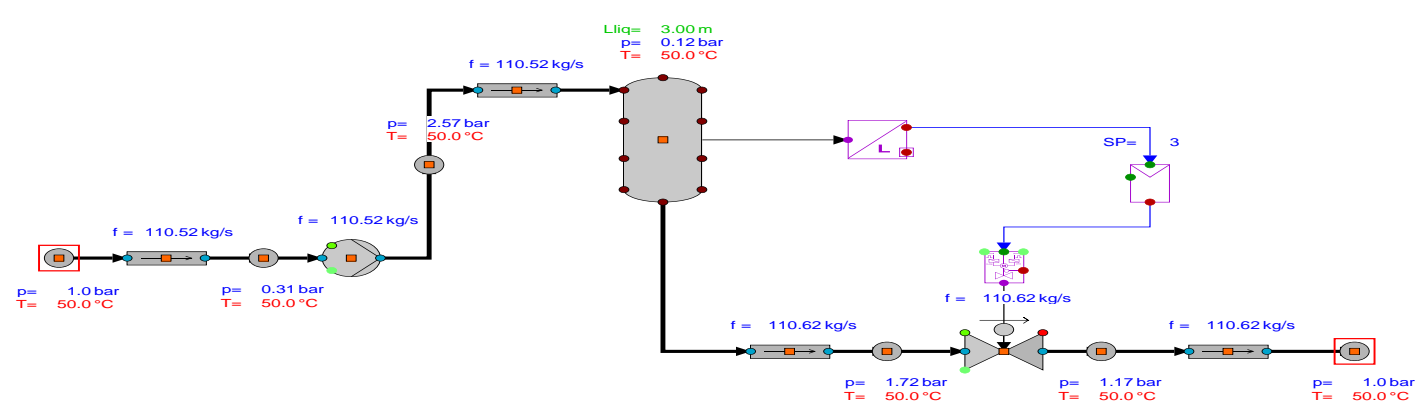

Figure 2. Apros model generated based on P\&ID illustrated in Figure 1.

more profitable in plant engineering by helping in discovering design flaws in an earlier phase.

Enhanced interoperability of engineering software based on standards enable additional use cases in addition to transferring data between corresponding design tools for continued design. In this paper, one such additional usage was exploited when the work conducted by the authors in generating dynamic simulation models based on ISO 15926-based P\&ID and 3D data in Proteus XML-format was reported. The features were implemented in dynamic process simulation software Apros and the Simantics platform.

The case study of the paper demonstrates successful utilisation of Proteus XML data for generating dynamic simulation models based on P\&ID data. Proteus is also capable of representing 3D data, enabling creation of more accurate simulation models, which has also been tried by the authors. Quality of the source data determines the accuracy achievable for the automatically generated simulation models. Therefore, the standard interfaces in engineering software used and the engineering practises applied in the engineering workflow shall support production of high quality data. The authors have tested CAE software from different vendors and concluded that the quality of standard interfaces varies and the interfaces need to become more mature to be effectively applicable in major industrial projects.

Utilising standard engineering data in engineering workflows is promising and a wider utilisation is demanded by the industry. One risk for a wider utilisation is whether the industry and software vendors can agree on a common standard since many promising alternatives currently exist. Therefore, co-operation between standardisation organisations, the industry and software vendors is required. Currently, availability and quality of interfaces vary but work conducted by e.g.
DEXPI show the interest for interoperability based on standards by industry, software vendors and academia.

Further validation is required for the implemented methods for automatic simulation model generation. The authors are going to validate the features in an extensive industrial use case to analyse the benefits and drawbacks more detailed. Simplification of simulation models by altering the nodalisation should also be handled in the automatic model generation.

\section{References}

M. Barth, M. Strube, A. Fay, P. Weber, and J. Greifeneder. Object-oriented engineering data exchange as a base for automatic generation of simulation models. In Annu Conf. IEEE Ind. Electron. Soc., Porto, 2009. doi: 10.1109/IECON.2009.5415229.

M. Barth and A. Fay. Automated generation of simulation models for control code tests. Control Eng. Practice, 21(2): 218-230, 2013. DOI:

10.1016/j.conengprac.2012.09.022.

buildingSMART International. IFC Overview summary. Updated: 2016. Accessed: 18.5.2016. Available: http://www.buildingsmart-tech.org/specifications/ifcoverview.

DEXPI. DEXPI - Data Exchange in the Process Industry. Updated 2016. Accessed 16.3.2016. Available: http://www.dexpi.org/.

E. Estevez and M. Marcos. Model-based validation of industrial control systems. IEEE Trans. Ind. Inform., 8(2): 302-310, 2012.

Fiatech. The Proteus project. Updated 2009. Accessed: 16.3.2016. Available:

http://fiatech.org/index.php/?option=com_content\&vie $\mathrm{w}=$ article\&id $=1115 \&$ Itemid $=748$.

Fiatech. ISO 15926 Information Models and Proteus Mappings IIMM. Updated 2015. Accessed 16.3.2016. Available: http://fiatech.org/information- 
management/projects/1161-iso-1592 6-informationmodels-and-proteus-mappings-iimm.

Fiatech and POSC Caesar Association. Proteus XML specification documents and XML schema. Updated: 2016. Accessed: 18.5.2016. Available: http://fiatech.org/information-management/ projects/1161iso-15926-information-models-and-proteusmappingsiimm.

Fortum and VTT Technical Research Centre of Finland. Apros Process Simulation Software. Updated: 2016. Accessed: 9.3.2016. Available: http://www.apros.fi/en/.

M. Hoernicke, A. Fay, and M. Barth. Virtual plants for brown-field projects. In IEEE Conf. Emerging Technol. Factory Autom., Luxembourg, 2015. doi: 10.1109/ETFA.2015.7301462.

T. Holm, L. Christiansen, M. Goring, T. Jager, and A. Fay. ISO 15926 vs. IEC 62424 - Comparison of plant structure modeling concepts. In IEEE Conf. Emerging Technol. Factory Autom., Krakow, 2012. doi:10.1109/ETFA.2012.6489662.

M. Hoyer, R. Schumann, and G. C. Premier. An approach for integrating process and control simulation into the plant engineering process. Comput. Aided Chemical Eng., 20(B): 1603-1608, 2005. doi: 10.1016/S15707946(05)80109-9.

International Electrotechnical Commission. IEC 62424:2008. Representation of process control engineering - Requests in P\&I diagrams and data exchange between P\&ID tools and PCE-CAE tools. 1st ed. 2008.

International Organization for Standardization. ISO 103031:1994. Industrial automation systems and integration Product data representation and exchange - Part 1: Overview and fundamental principles. 1st ed. 1994.

International Organization for Standardization. ISO 159261:2004. Industrial automation systems and integration Integration of life-cycle data for process plants including oil and gas production facilities - Part 1: Overview and fundamental principles. 1st ed. 2004.

International Organization for Standardization. ISO 16739:2013. Industry Foundation Classes IFC for data sharing in the construction and facility management industries. 1st ed. 2013.

T. Karhela, A. Villberg, and H. Niemistö. Open ontologybased integration platform for modeling and simulation in engineering. Int. J. Modeling, Simulation, and Scientific Computing, 3(2): 1250004-1-1250004-36, 2012.

P. Laakso, J. Lappalainen, T. Karhela, and M. Luukkainen. Virtual plant combines engineering tools for the process industry. VTT Research Highlights 8, 2013. Accessed: 11.5.2016. Available: http://www.vtt.fi/inf/pdf/researchhighlights/ 2013/R8.pdf.

Nextspace. XMpLant. Updated 2015. Accessed: 16.12.2015. Available: http://www.nextspace.co.nz/products-andservices/solutions/xmplant/.

Open CASCADE. Open CASCADE Technology. Updated: 2016. Accessed 16.5.2016. Available: http://www.opencascade.com/.

M. Oppelt and L. Urbas. Integrated virtual commissioning an essential activity in the automation engineering process: From virtual commissioning to simulation supported engineering. In Annu. Conf. IEEE Ind. Electron. Soc., Dallas, TX, 2014. doi: 10.1109/IECON.2014.7048867.

M. Oppelt, M. Barth, and L. Urbas. The Role of Simulation within the Life-Cycle of a Process Plant. Results of an online survey, 2015a. doi: 10.13140/2.1.2620.7523.

M. Oppelt, G. Wolf, and L. Urbas. Towards an integrated use of simulation within the life-cycle of a process plant. In IEEE Conf. Emerging Technol. Factory Autom., Luxembourg, 2015b. doi: 10.1109/ETFA.2015.7301521.

Oracle. Java 2D Graphics and Imaging. Updated: 2016.

Accessed: 18.5.2016. Available: https://docs.oracle.com/javase/8/docs/techn otes/guides/2d/.

N. Paganus, K. Honkoila, and T. Karhela. Integrating dynamic process simulation into detailed automation engineering. In 21st IEEE International Conference on Emerging Technology and Factory Automation ETFA'2016, 6-9.9.2016, Berlin, Germany .

M. Paljakka, J. Talsi, and H. Olia. Experiences on the integration of automation CAE and process simulation tools - case FupRos. In Automaatio XVIII Seminar, Finnish Society of Automation, publication series 36, Helsinki, Finland, 2009.

K. Porkholm, H. Kontio, H. Plit, M. Mustonen, and K. Söderholm. APROS simulation model for Olkiluoto-3 EPR Applications. In European Nuclear Society TopSafe Trans., Dubrovnik, Croatia, 2008. Accessed: 9.3.2016. Available: http://www.euronuclear.org/events/topsafe/transactions/To pSafe2008-transactions-poster.pdf.

SAX. SAX - Simple API for XML. Updated: 2004. Accessed: 18.5.2016. Available: http://sax.sourceforge.net/.

Simantics. Open operating system for modeling and simulation. Updated: 2016. Accessed: 14.4.2016. Available: https://www.simantics.org/.

Eclipse. The Eclipse Foundation open source community website. Updated 2016. Accessed 16.5.2016. Available: http://www.eclipse.org/.

THTH. THTH Association of Decentralized Information Management for Industry. Updated: 2016. Accessed: 18.5.2016. Available: http://www.ththry.org/?lang=en.

G. P. Towler and R. K. Sinnott. Chemical Engineering Design: Principles, Practice, and Economics of Plant and Process Design. 2nd ed. Oxford, UK, ButterworthHeinemann, 2013.

A. Tursi, H. Panetto, G. Morel, and M. Dassisti. Ontological approach for products-centric information system interoperability in networked manufacturing enterprises. Annual Reviews in Control, 33(2): 238-245, 2009. DOI: 10.1016/j.arcontrol.2009.05.003.

VTK. VTK - The Visualization Toolkit. Updated: 2016. Accessed 16.5.2016. Available: http://www.vtk.org/.

A. Wiesner, J. Morbach, and W. Marquardt. Information integration in chemical process engineering based on semantic technologies. Computers \& Chemical Engineering, 35(4): 692-708, 2011. DOI: 10.1016/j.compchemeng.2010.12.003. 